\title{
Electronic nose system for rancidity and insect monitoring of brown rice
}

\author{
Natawut Neamsorn ${ }^{1,2^{*}}$, Viboon Changrue ${ }^{1,2}$, Yaowalak Chanbang ${ }^{1,3}$, \\ Kaewalin Kunasakdakul ${ }^{1,3}$, Parichat Theanjumpol ${ }^{1,3}$, and Phongprapan Kantakaew ${ }^{1,2}$ \\ ${ }^{1}$ Ministry of Higher Education, Science, Research and Innovation, Postharvest Technology \\ Innovation Center, 238 Thanon Si Ayutthaya, Ratchathewi, Bangkok 10400, Thailand \\ ${ }^{2}$ Chiang Mai University, Faculty of Engineering, Chiang Mai 50200, Thailand \\ ${ }^{3}$ Chiang Mai University, Faculty of Agriculture, Chiang Mai 50200, Thailand
}

\begin{abstract}
Electronic nose system was designed and fabricated for classification of rancidity and pest damages in brown rice. The electronic nose system was included gas handling system, sensors array and data acquisition and processing system. Response signal from sensors array was recorded and processed. The results showed that the E-nose could classify normal and rancid brown rice (KDML105) and the classification model had $\mathrm{R}_{\mathrm{v}}{ }^{2}=0.92$ and SEP $=0.14$. The model also gave satisfactory result for classification of brown rice which was damaged by insects (Tribolium castaneum) with $\mathrm{R}_{\mathrm{v}}{ }^{2}=0.98$ and $\mathrm{SEP}=0.06$. It was possible to use electronic nose as the quality monitoring system during storage of KDML105 brown rice.
\end{abstract}

\section{Introduction}

Rice is one of major food staples with the importance equal to wheat and grown in more than 100 countries around world [1]. Brown rice, which is whole grain rice, is produced by dehusking the paddy (rough rice). Compared with white rice (milled rice), brown rice has more nutrients content e.g., Thiamine, oil content, fiber, niacin, phosphorus, potassium, iron, sodium and riboflavin [2]. Because of the health benefits, doctors encourage their patients to consume more brown rice [3]. However, during storage, brown rice is degraded due to the internal chemical and physical changing and also insect damage. The traditional measurements are not able to detect the degrading in early stage.

Electronic nose is designed for odor detection and discrimination by mimicking the mammal olfaction system. The basic components of electronic nose include handling system, sensor arrays and processing systems [4]. The electronic nose is inexpensive, portable and able to rapidly detect complex volatile mixtures. Furthermore, the electronic nose can detect odourless compounds which cannot be smelled by human [5]. Nowadays, electronic nose widely applies in different sectors of agriculture, for example food and beverage processing, quality control, crop protection, etc. However, there are limited information of electronic

\footnotetext{
* Corresponding author: natawut.neamsorn@emu.ac.th
} 
nose for brown rice quality monitoring application. Therefore, this research studied the possibility of detecting degraded brown rice during storage using an electronic nose system.

\section{Materials and methods}

\subsection{Electronic nose system}

The electronic nose system was designed and fabricated in the agricultural engineering laboratory, Chiang Mai university. The schematic diagram of the system showed in Figure 1. The environmental air flowed through filters by micro-pump at a flow rate of $0.4 \mathrm{~L} / \mathrm{min}$, which acquired from preliminary test. Three solenoid valves were used as air directional control valves. A personal computer controlled the system, transferred and collected the response signal data via a LabVIEW data acquisition hardware, NI-USB 6009. The sensors array, which were metal oxide based (MOS) gas sensors, was installed as shown in the series layout (Figure 2). The eight MOS gas sensors (i.e., TGS822, TGS826, TGS2600, TGS2610, TGS2620, MQ3, MQ7, MQ8) were in a sealed acrylic glass container. The list of target gases, which were suggested by the manufacturer, were shown in Table 1.

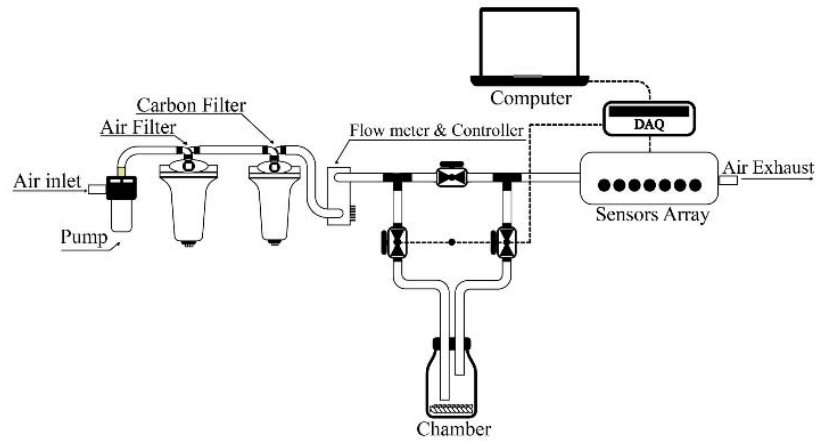

Fig. 1. Schematic diagram of electronic nose system.

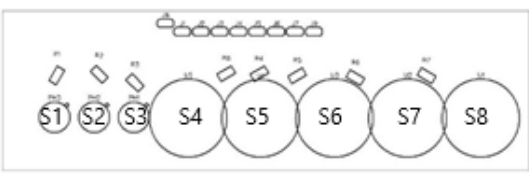

Fig. 2. Sensor arrays layout. 
Table 1. List of target gases for the sensors.

\begin{tabular}{|c|c|}
\hline Sensor type & Target gases \\
\hline TGS 822 & organic solvent vapors \\
\hline TGS 826 & ammonia \\
\hline TGS 2600 & $\begin{array}{c}\text { air contaminants } \\
\text { (hydrogen, ethanol, etc.) }\end{array}$ \\
\hline TGS 2610 & butane, propene \\
\hline TGS 2620 & alcohol, solvent vapors \\
\hline MQ 3 & alcohol \\
\hline MQ 7 & carbon monoxide \\
\hline MQ 8 & hydrogen \\
\hline
\end{tabular}

\subsection{Rancid brown rice}

KMDL 105 paddy was obtained from local farmer in Chiang Mai province. The paddy was cleaned by a winnower and de-husked by a rubber roll husker. The un-husked paddy was removed from brown rice by a paddy separator. Finally, broken brown rice was removed by a rice grader. Fifty-gram samples of rancid and normal brown rice with moisture content of $11.4 \%$ were kept in $250 \mathrm{ml}$ sealed glass bottles and stored in a cold and dark room. The storage temperature was $8^{\circ} \mathrm{C}$. Before the experiment, the glass bottles were placed at room temperature for $20 \mathrm{~min}$.

\subsection{Insect damaged brown rice}

The normal brown rice was prepared in the same manner as the previous experiment and the moisture content of brown rice was $11.4 \%$. Fifty-gram samples of brown rice contained in $250 \mathrm{ml}$ glass bottles with 20 adult red flour beetles (Tribolium castaneum) and kept at room temperature for 7 days.

\subsection{Electronic nose measurement}

The normal, rancid and insect damaged brown rice, which were in the chamber, was connect to the electronic nose system and measuring with 200 repetitions. Each measurement was performed in 3 steps; flushing (60 s), generating (20 min) and measuring (90 s). The measuring data were collected and recorded via a personal computer with $1 \mathrm{kHz}$ of sampling frequency before analysed by using the principle component analysis (PCA) and partial least square discriminant analysis (PLS-DA). In the model building, 266 samples were use as model calibration and 133 sample. were uses as model calibration. 


\section{Results and discussion}

\subsection{Electronic nose response}

The signal received from electronic nose system while measuring was voltage signal variation with time (Figure 3 ). When the odor exposed to the sensors, voltage signal increased from baseline for the initial stage. The signal gradually decreased in the situated stage due to odor concentration change. In the final stage, when the odor was diverted from the sensors array, the signal was decreased continuously to the baseline signal.

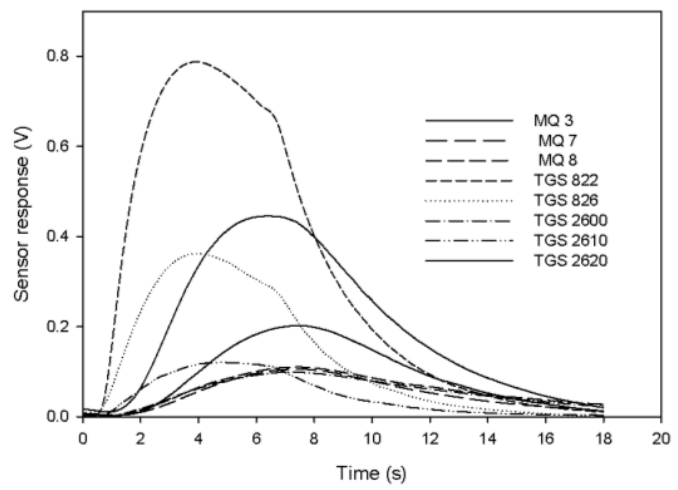

Fig. 3. Electronic nose signal response.

\subsection{Rancid and insect damaged brown rice measurement}

The area under curve during measuring time was the response data analysed by principle component analysis (PCA) technique. The score plot of PCA for normal brown rice and rancid brown rice and for normal brown rice and insect damaged brown rice was shown in figure 4 . There were two clusters formed clearly in the plots. The first cluster corresponded to normal brown rice while the second cluster corresponded to rancid brown rice (Figure 4 (a)) and insect damaged brown rice (Figure 4 (b)). For the rancid and insect damaged brown rice, the $\mathrm{PC}$ was $77 \%$ and $99 \%$ respectively.

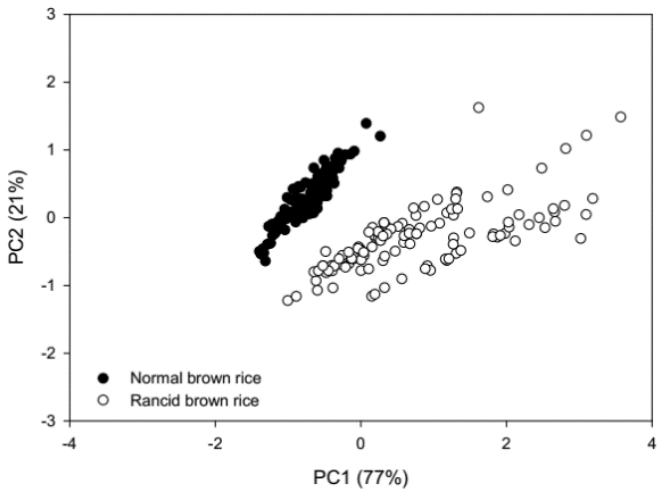

(a)

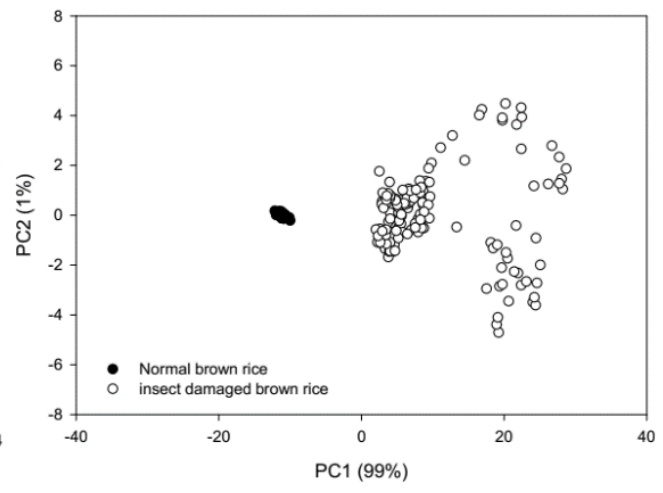

(b)

Fig. 4. Score plot of PCA for rancid brown rice (a) and insect damaged brown rice (b). 
The PLS-DA models were developed to test the classification performance of electronic nose systems. In validation test, the PLS-DA models gave satisfactory result for classification of rancidity brown rice $\left(\mathrm{R}_{\mathrm{v}}{ }^{2}=0.92, \mathrm{SEP}=0.14\right)$. and insect damaged brown rice $\left(\mathrm{R}_{\mathrm{v}}{ }^{2}=0.98\right.$, $\mathrm{SEP}=0.06)$.

\section{Conclusion}

The electronic nose based on array of MOS gas sensors was the rapid instrument for rancidity and insect damage of brown rice detection. The signal response correlated with brown rice classification (normal, rancid and insect damage). The electronic nose system is potential application for quality control and rancidity check during storage in the rice industry.

This research is supported by Postharvest Technology Innovation Center, Ministry of Higher Education, Science, Research and Innovation, Bangkok, Thailand.

\section{References}

1. C.W. Smith, R.H. Dilday, Rice: Origin, History, Technology, and Production: Wiley (2002)

2. W.E. Marshall, J.I. Wadsworth, Rice Science and Technology: Taylor \& Francis (1993)

3. S.A.Mir, A. Manickavasagan, M.A. Shah, Whole Grains: Processing, Product Development, and Nutritional Aspects: CRC Press (2019)

4. T.C. Pearce, S.S. Schiffman, H.T. Nagle, J.W. Gardner, Handbook of Machine Olfaction: Electronic Nose Technology: Wiley (2006)

5. H.K. Patel, The Electronic Nose: Artificial Olfaction Technology: Springer India (2013) 